\title{
Conformal Villarceau Rotors
}

\author{
Leo Dorst* (1)
}

\begin{abstract}
We consider Villarceau circles as the orbits of specific composite rotors in 3D conformal geometric algebra that generate knots on nested tori. We compute the conformal parametrization of these circular orbits by giving an equivalent, position-dependent simple rotor that generates the same parametric track for a given point. This allows compact derivation of the quantitative symmetry properties of the Villarceau circles. We briefly derive their role in the Hopf fibration and as stereographic images of isoclinic rotations on a 3 -sphere of the $4 \mathrm{D}$ Clifford torus. We use the CGA description to generate 3D images of our results, by means of GAviewer. This paper was motivated by the hope that the compact coordinate-free CGA representations can aid in the analysis of Villarceau circles (and torus knots) as occurring in the Maxwell and Dirac equations.
\end{abstract}

Mathematics Subject Classification. Primary 51Bxx; Secondary 15A66.

Keywords. Villarceau circle, Conformal, Geometric algebra, Hopf fibration, Isoclinic rotation, Clifford torus.

\section{Villarceau Circles}

In two recent papers, by Consa [3] and Hestenes [8], models of the electron are proposed that involve it moving rapidly internally around a torus. The parametrization of that motion in both papers is done by the two uniformly increasing angles evolving from the usual Euclidean image of generating a torus as a circle moving around a circle. In [9], Hestenes derives this form explicitly as a solution of the Dirac equation when a Lorentz boost is factored out.

A second physical situation in which orbits on a torus occur is in the knotted solutions to Maxwell's equation in vacuum, in which the Poynting vector of the electromagnetic field lies on a torus knot [2]. Such a knot is a

This article is part of the Topical Collection on Proceedings of AGACSE 2018, IMECCUNICAMP, Campinas, Brazil, edited by Sebastià Xambó-Descamps and Carlile Lavor.

*Corresponding author. 


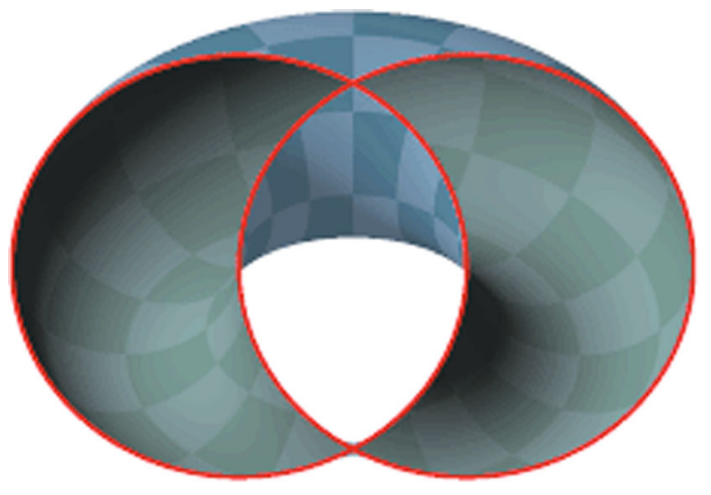

FiguRE 1. Villarceau circles visible on a cut torus, from [13]

geometric object, not a path, and it no longer requires a parametrization to describe its time dependence - the field lines themselves evolve with the speed of light. Any convenient parametrization works, though the innate conformal symmetry of the Maxwell equation may prefer a conformal parametrization.

In both applications, the simplest orbits or field lines are the $(1,1)$ torus knots. In this paper, we analyze their remarkable properties (they are circles!) by means of our representation from [5], where we encountered $(m, n)$ torus knots as the orbits of certain conformal rotors. The natural parametrization of that solution was based on an orthogonal (commuting) decomposition, which involves one Euclidean rotation around the torus axis, and a 'conformal rotation' in the orthogonal direction. The resulting motion differs intriguingly from the Consa/Hestenes parametrization, and the canonical orbits of the two parametrizations are not identical.

In mathematics, the path that the $(1,1)$ knot on the torus traces is also known as a Villarceau circle. ${ }^{1}$ Villarceau circles are usually introduced as two intersecting circles that are the cross-section of a torus by a well-chosen plane cutting it (Fig. 1). Picking one such circle and rotating it around the torus axis, the resulting family of circles can be used to rule the torus. By nesting tori smartly, the collection of all such circles then form a Hopf fibration of 3D space. In the theory of Penrose twistors, the Villarceau circles occur as the Robinson congruence, the family of light rays representing a non-null twistor; this is yet another link to interesting physics (see e.g. [1] for a geometric algebra treatment).

In the present paper, we prefer to consider the Villarceau circle as the $(1,1)$ torus knot rather than as a planar cut, and we generate it by means of a composite rotor in conformal geometric algebra in Sect. 2. We then characterize any Villarceau circle compactly as the orbit of a location-dependent simple rotor (after studying those in detail in Sect. 3) passing through a given

\footnotetext{
${ }^{1}$ As yet another example of a concept named for somebody who did not invent it, Villarceau circles were apparently found by a Colonel Schölcher in 1891, though some of their conformal properties were shown by Villarceau in 1903 (says Wikipedia [13]).
} 
point, with a varying speed determined by this rotor (Sect. 4). That compact description permits the derivation of a number of geometric properties (including the planar cut construction), many involving a specific imaginary sphere (Sect. 5), which is a natural completion of the conformal basis. The Hopf fibration then follows simply from letting the rotor act on arbitrary points, and by means of spherical inversion we can relate this directly to isoclinic rotations on the Clifford torus in 4D (in Sect. 6). The conformal nature of the construction allows a straightforward extension to Villarceau circles on arbitrary Dupin cyclides, in Sect. 7.

\section{Rotor Generation}

In [5] we showed how 3D conformal motions can be generated as the orbit of a rotor that is the exponential of the sum of two commuting 2-blades of CGA. These 2-blades geometrically represent 'orthogonal' point pairs (with perpendicularity properties described that paper). In the case of the torus, one point pair is the dual of a line, the other an imaginary point pair on that line. Both point pairs have a negative square, so that each generates periodic orbits.

As our prototype for this situation we can take $B_{-}=\mathbf{e}_{1} \mathbf{e}_{2}$ and $B_{+}=$ $\mathbf{e}_{3} e_{+}$, in the usual orthonormal basis $\left\{\mathbf{e}_{1}, \mathbf{e}_{2}, \mathbf{e}_{3}, e_{+}, e_{-}\right\}$of the $\mathbb{R}^{4,1}$ Minkowski space for the description of $3 \mathrm{D}$ conformal geometric algebra. Boldly denoted are the basis vectors for the Euclidean space (which can be interpreted as orthogonal planes at the origin), and $e_{+}$and $e_{-}$are the unit real sphere and the unit imaginary sphere, respectively. So $e_{-}^{2}=-1$, all other orthogonal basis vectors square to +1 . For each of the 'real' (positively squared) basis elements, algebraic orthogonality is equivalent to geometric perpendicularity of the corresponding geometrical primitives at their intersection. For the 'imaginary' sphere, algebraic orthogonality with the real planes and the real sphere geometrically implies that the corresponding intersection should be an equator of the imaginary sphere (see [4] Chapter 14).

We can construct 2-blades from these basis vectors, and exponentiate them, as a canonical way of making rotors. Three different types of rotor result, depending on the sign of the square of the 2-blade. We illustrate the orbits of a point moving by each of them in Fig. 2. When the square is positive, the orbit has a source and a sink; when negative, it is periodic; when zero (for a blade containing a null vector like $o=e_{-}+e_{+}$or $\left.\infty=e_{-}-e_{+}\right)$, merely a sink. We will analyze these cases and their parametrizations in more detail in Sect. 3.

Since $B_{-}=\mathbf{e}_{1} \wedge \mathbf{e}_{2}$, with $B_{-}^{2}=-1$, the simple rotor $R_{-}=\exp \left(-B_{-} \phi / 2\right)$ generates periodic circular orbits $x(\phi)=R_{-} x / R_{-}$around the axis $\left(B_{-}\right)^{*}$, the dual of the 2 -blade $B_{-}$. For any point $x$, its orbit is the circle $x \wedge B_{-}$ in the plane $x \wedge B_{-} \wedge \infty$. At a given point $x$, the other simple rotor $R_{+}=$ $\exp \left(-B_{+} \psi / 2\right)$, with $B_{+}=\mathbf{e}_{3} \wedge e_{+}$, also satisfying $B_{+}^{2}=-1$, generates a circle $x \wedge B_{+}$, which is in the plane $x \wedge B_{+} \wedge \infty$. These $B_{+}$-circles shrink as the point $x$ gets closer to their 'core', i.e. the circle $\left(B_{+}\right)^{*}$ which is the 


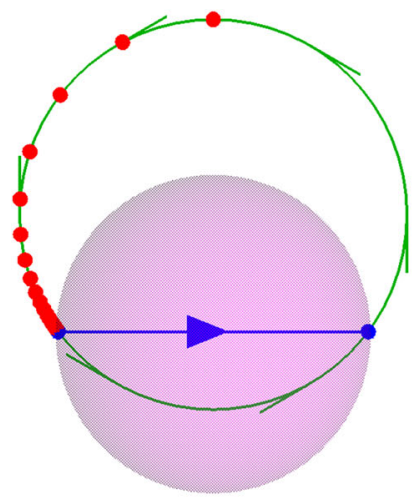

(a)

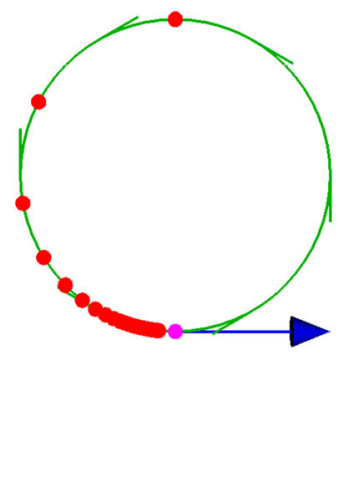

(b)

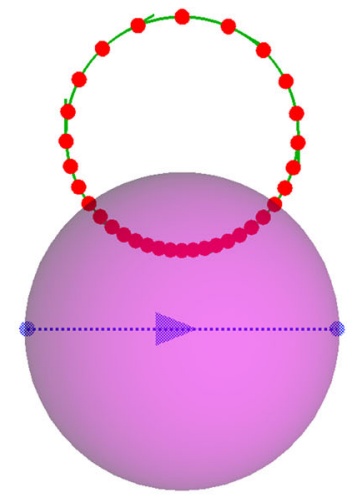

(c)

FiguRE 2. The orbits generated by simple rotors, the exponentials of $\mathbf{a}$ a real point pair (such as $\mathbf{e}_{3} e_{-}$), $\mathbf{b}$ a null point pair (such as $\mathbf{e}_{3}\left(e_{+}+e_{-}\right) / 2=\mathbf{e}_{3} o$ ) and $\mathbf{c}$ an imaginary point pair (such as $\mathbf{e}_{3} e_{+}$). Equal increments of the scalar parameter of the 2-blade arguments generate subsequent points, along circular orbits. The spheres denote the 'surrounds' of the point pair (the smallest sphere of which it is a pair of antipodal points). These figures are planar, residing in the plane determined by the starting point and the point pair; but they can be viewed as cross sections of a $3 \mathrm{D}$ pattern obtained by rotation around the point pair axis

intersection of the dual plane $\mathbf{e}_{3}$ with the dual unit sphere $e_{+}$. This 'core' is not the Euclidean center of the circles, but acts as their conformal center.

The simple rotors $R_{-}$and $R_{+}$can be composed into the product rotor

$$
\begin{aligned}
R & =R_{+} R_{-}=R_{-} R_{+} \\
& =\exp \left(-B_{-} \psi / 2\right) \exp \left(-B_{+} \phi / 2\right) \\
& =\exp \left(-B_{-} \psi / 2-B_{+} \phi / 2\right),
\end{aligned}
$$

where the addition of the exponent is permitted since the 2-blades commute. When moving in the product of the rotors, the resulting orbit resides on a torus, see [5]. When $\phi$ and $\psi$ are taken in a ratio of $\psi= \pm(m / n) \phi$, the orbit is a periodic $(m, n)$ torus knot. These knots are inscribed on the torus in a conformal manner, cutting all $B_{+}$-circles of longitude (or $B_{-}$-circles of latitude) by equal angles. This is illustrated in Fig. 3a (from [5]), which shows the $R$ rotor orbit in red, and also indicates the $R_{-}$-orbit (in blue) and the $R_{+}$-orbit (in green). The knotted red orbit can be seen as locally composed of small steps in each of those circular orbits.

To obtain a Villarceau circle through a point $x$, we choose $\psi= \pm \phi$, generating a $(1,1)$ torus knot depicted in Fig. 3b. Let us define $B$ as the sum of the unit 2-blades:

$$
B=B_{-}+B_{+} .
$$




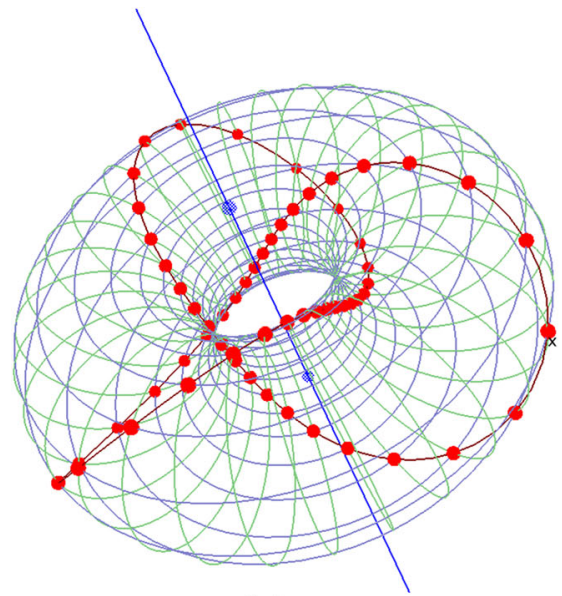

(a)

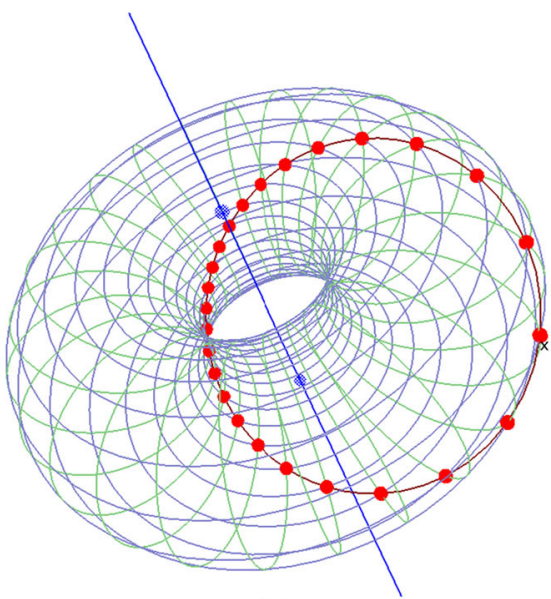

(b)

Figure 3. a The $(2,3)$ torus knot generated by a conformal rotor. $\mathbf{b}$ The $(1,1)$ toruskot, a Villarceau circle. In both, the blue circles are local orbits of $R_{-}$, the green circles are local orbits of $R_{+}$. The generator 2-blade of $R_{-}$is the blue line, the generator 2-blade of $R_{+}$the imaginary point pair (color figure online)

This $B$ is not a 2-blade (its square $B^{2}=B_{-}^{2}+B_{+}^{2}+2 B_{-} \wedge B_{+}$is not a scalar), so it cannot be factored by the outer product. Now form a rotor by exponentiation of $B$, apply it to a point $x$ :

$$
x(\phi)=e^{-\phi B / 2} x e^{\phi B / 2},
$$

and investigate its orbit - which should be a Villarceau circle. But this is not obvious! The orbit of a simple conformal rotor based on a 2-blade rotor is always a circle, as we illustrated in Fig. 2 (with a line, the orbit of a translation rotor, considered as a circle passing through infinity). The orbit of a rotor based on a general 2-vector, such as $B$ above, is not. To demonstrate that in this exceptional case we still obtain a circle, we show that its orbit $x \wedge B$ is identical to that of a 2-blade (which will depend on $x$ ) - even though $B$ is not a 2-blade itself. Before we can do so, we first need to expose the simple rotors and their parametrizations in more detail.

\section{Orbits of Simple Conformal Rotors}

To analyze the orbit of the non-simple rotor above, let us briefly derive the properties and parametrization of simple rotors (i.e., exponentials of 2-blades) in conformal geometric algebra. Our main focus is on the exponential of an 'imaginary' 2-blade (i.e. one that squares to a negative scalar). But while we are at it, we can include real and null 2-blades with very little additional effort, even though their characteristics differ considerably (as Fig. 2 showed). 


\subsection{Imaginary Point Pair: Periodic Motions}

Consider an imaginary point pair $P$ (a 2-blade from CGA), normalized so that $P^{2}=-1$. For invertible $P$, we can split $x$ into its projection and rejection relative to $P$, and write

$$
\begin{aligned}
x(\phi) & \equiv e^{-\phi P / 2} x e^{\phi P / 2} \\
& =e^{-\phi P / 2}((x \cdot P) / P+(x \wedge P) / P) e^{\phi P / 2} \\
& =(x \wedge P) / P+(x \cdot P) / P e^{\phi P}
\end{aligned}
$$

using commutation and anti-commutation properties of those split terms with the $P$-rotor. For an imaginary point pair, the exponential involves trigonometric functions: $\exp (\phi P)=\cos (\phi)+P \sin (\phi)$. Using those, $x(\phi)$ can be expanded to:

$$
x(\phi)=(x \wedge P) / P+(x \cdot P) / P \cos \phi+(x \cdot P) \sin \phi .
$$

The vectors $(x \cdot P),(x \cdot P) / P$ and $(x \wedge P) / P$ form an orthogonal basis for the orbit, consisting of two real spheres $(x \cdot P)$ and $(x \cdot P) / P$, and an imaginary sphere $(x \wedge P) / P$ (intersected by the real spheres in its equator). So using CGA, we can describe a moving point on a circle as the weighted sum of three specific orthogonal spheres.

The parametrization by $\phi$ of the points along the circle is not-equidistant, but equiangular in a conformal manner. Since all the terms in Eq. (3) transform covariantly under conformal transformations, we can design a conformal mapping from a canonical situation that is particularly intuitive to parametrize, and then use that parametrization for the general case. Let us explain this by a specific example, illustrated in Fig. 4a, b.

The Euclidean rotation with rotation rotor determined by the 2-blade $P=\mathbf{e}_{1} \mathbf{e}_{2}$ has a natural equi-angular parametrization relative to the center, the origin indicated by the conformal point vector $o$. The transformed points $x(\phi)=\exp (-\phi P / 2) x \exp (\phi P / 2)$ along the orbit $x \wedge P$ then lie on rays $o \wedge$ $x(\phi) \wedge \infty$, and the angular parameter makes the points traverse this orbit at a uniform speed.

Now transforming this situation by inversion in $\sigma=\mathbf{e}_{2}+e_{+}$, the 2blade becomes $\mathbf{e}_{1} e_{+}$, and its rotor represents a 'conformal rotation'. The center $o$ transforms to $-\sigma o / \sigma=\left(\mathbf{e}_{2}-e_{-}\right) / 2=-\frac{1}{2} \mathrm{CGA}\left(-\mathbf{e}_{2}\right)$, i.e. the point at location $-\mathbf{e}_{2}$ (with weight $-\frac{1}{2}$ ), and the point at infinity $\infty$ becomes the finite point $-\sigma \infty / \sigma=-\mathbf{e}_{2}-\mathbf{e}_{-}=-\operatorname{CGA}\left(\mathbf{e}_{2}\right)$, i.e. the point at $\mathbf{e}_{2}$ (with weight -1). As a consequence of this transformation of $o$ and $\infty$, the equiangular rays $o \wedge x(\phi) \wedge \infty$ of the Euclidean equi-angular parametrization become circles, still equi-angular relative to the new center $-\sigma o / \sigma$ (since the inversion is conformal). Intersections also transform covariantly, so where the circle with angle $\phi$ cuts the new orbit is where the transformed parametrized point $x^{\prime}(\phi)=-\sigma x(\phi) / \sigma$ ends up.

This covariant relationship is illustrated in Fig. 4b, which shows how the Euclidean situation of Fig. 4a is conformally transformed by inversion in $\sigma$. Our figure is effectively a re-rendering of Picture 27 in Chapter 6 of the enjoyable book by Needham [10] in Visual Complex Analysis (though we do 


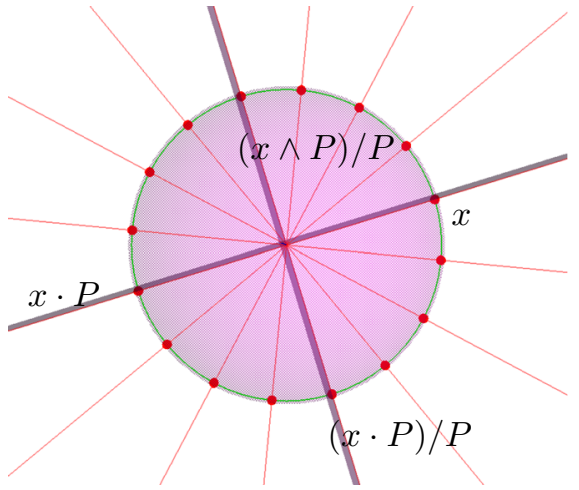

(a)

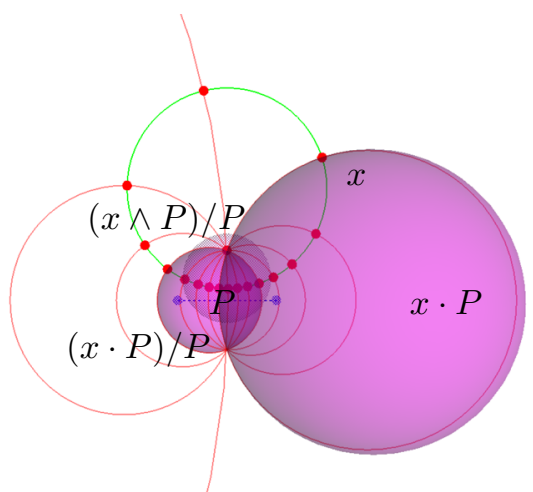

(b)

Figure 4. a A Euclidean rotation, b a conformal rotation, obtained from a by an inversion. Equiangular rays from the conformal center are indicated in red. In $\mathbf{b},(x \cdot P) / P$ is an imaginary sphere, depicted as dotted (color figure online)

not use complex numbers, and therefore are not limited to 2D). Figure 4b also illustrates how the orthogonal basis of Fig. 4a gets transformed in an orthogonal basis for the transformed circle. The imaginary sphere $e_{-}$remains, the planes through $x$ and the plane perpendicular to that become orthogonal spheres (still cutting the imaginary sphere $e_{-}$in great circles).

This particular example is the typical situation: we can always design a conformal transformation to transform the Euclidean axis 2-blade to a desired conformal 2-blade, and the Euclidean equiangular parametrization then transforms to the equiangular parametrization from the transformed center. To transform $\mathbf{e}_{1} \mathbf{e}_{2}$ to the product of two arbitrary orthogonal unitsquare spheres $\sigma_{1} \sigma_{2}$, one may use the method from [6] to construct the corresponding transformation versor as $\left(\sigma_{1}-\mathbf{e}_{1}\right) \mathbf{e}_{2}+\sigma_{2}\left(\sigma_{1}-\mathbf{e}_{1}\right)$.

\subsection{A Real Point Pair: Exponential Motion from Source to Sink}

The derivation of Eq. (2) also holds for a real point pair, where $P^{2}=1$. The expansion now involves hyperbolic functions; substituting $\exp (\gamma P)=$ $\cosh (\gamma)+P \sinh (\gamma)$ we obtain

$$
x(\gamma)=(x \wedge P) / P+(x \cdot P) / P \cosh \gamma+(x \cdot P) \sinh \gamma .
$$

Again the moving point is a weighted sum of elements $(x \cdot P),(x \cdot P) / P$ and $(x \wedge P) / P$, which form an orthogonal basis of three orthogonal spheres. See Fig. 5b.

For the standard situation, we can actually use the same inversion as for the rotation, but now considering the radial motions. Figure 5a shows an exponential scaling relative to the flat center $o \wedge \infty=e_{+} \wedge e_{-}$. The 2-blade for the radial rotor $\exp (-\gamma o \wedge \infty / 2)$ transforms any point on a radial ray to a scaled location which is $e^{-\gamma}$ of the previous distance of the center (simultaneously affecting its weight by a factor $e^{\gamma}$ ). This rotor therefore provides 


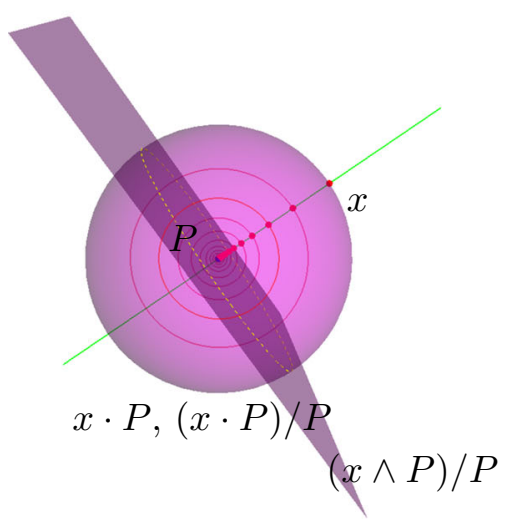

(a)

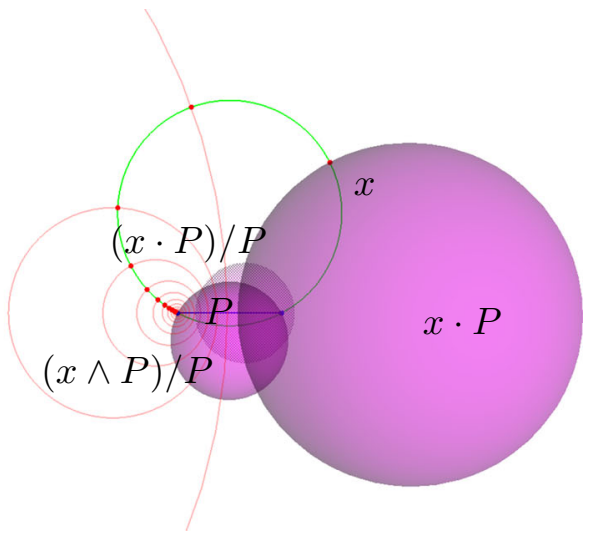

(b)

Figure 5. a A Euclidean exponential scaling: $P$ is a flat point, and the real and imaginary spheres $x \cdot P$ and $(x$. $P) / P$ coincide. $\mathbf{b}$ A conformal scaling, obtained from a by an inversion. Circles of equal logarithmic increase are indicated in red (color figure online)

a natural scaling parameter in the radial direction. The exponential allows the full range of $\gamma \in(-\infty, \infty)$ without leading to awkwardness at the (unreachable) origin, and therefore this parametrization by $\gamma$ is more natural than the customary linear radial parameter of polar coordinates. Applying the inversion in the sphere $\sigma=\mathbf{e}_{2}+e_{+}$, the 2-blade $e_{+} \wedge e_{-}$becomes the real point pair $\mathbf{e}_{2} \wedge e_{-}$, and the radial motions become circular motions on circles emanating from the transformed origin. Thus Fig. 5a is transformed into Fig. 5b. Yet the parameter for 'natural' motion along the orbits of the transformed rotor $\exp \left(-\gamma \mathbf{e}_{2} \wedge e_{-}\right)$is still $\gamma$, to produce the transformed point $-\sigma x(\gamma) / \sigma$. That moving point departs from the source, and approaches the sink, in a hyperbolic pacing along the circular path, by Eq. (4).

\subsection{A Null Point Pair: Translations and Tranversion}

For the noninvertible case $P^{2}=0$, Eq. (2) does not apply. Instead, we can just expand the series of Eq. $(6)$, using $(x \cdot P) \cdot P=(x \cdot P) P,((x \cdot P) \cdot P) \cdot P=$ $(x \cdot P) P^{2}=0$. That yields $x(\alpha)=x+\alpha(x \cdot P)\left(1+\frac{1}{2} \alpha P\right)$, which may be regrouped to:

$$
x(\alpha) \equiv e^{-\alpha P / 2} x e^{\alpha P / 2}=x+\alpha(x \cdot P) \exp (\alpha P / 2) .
$$

Since $x \cdot((x \cdot P) P)=-(x \cdot P)^{2} \neq 0$, the basis formed by $x, x \cdot P$ and $(x \cdot P) P$ is not orthogonal.

For null blades, the prototypical Euclidean situation is a translation with 2 -blade $-\alpha \mathbf{e}_{1} \infty / 2$. Orthogonal to its orbit (a straight line) are the hyperplanes with normal vector $\mathbf{e}_{1}$, equally spaced as $\mathbf{e}_{1}+\alpha \infty$, see Fig. 6 a.

An inversion in the origin sphere $e_{+}$converts this situation to the 2-blade $\alpha o \mathbf{e}_{1}$, which characterizes a transversion (in Fig. 6b), and the equidistant 


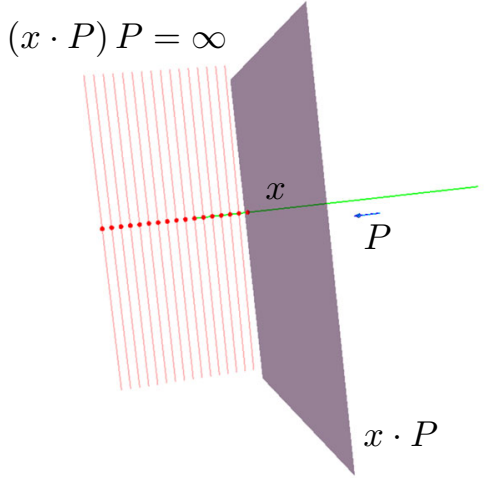

(a)

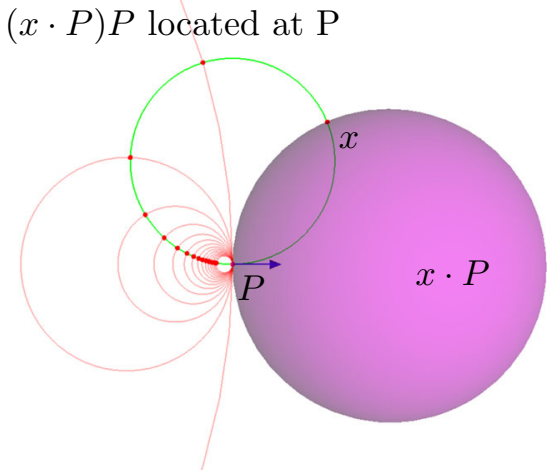

(b)

Figure 6. a A Euclidean translation, b a conformal transversion, obtained from a by an inversion

planes $\mathbf{e}_{1}+\alpha \infty$ now become the spheres $\mathbf{e}_{1}+2 \alpha o$ passing through $o$ with center $\mathbf{e}_{1} /(2 \alpha)$, which cut the new orbit $x^{\prime} \wedge o \wedge \mathbf{e}_{1}$ at the $\alpha$-parametrized point $x^{\prime}(\alpha)$. Uniform translational speed towards infinity in the Euclidean situation naturally becomes a convergence to the sink of the transversion, by circular motions whose angle relative to the Euclidean center of the circular orbit can be shown to vary as the arctangent of the reciprocal of $\alpha$.

\section{Simplifying the Villarceau Rotor}

To resume our tale after Eq. (1), we look for a simple rotor (i.e. the exponential of a 2-blade) of which the orbit through $x$ is the same as that of $\exp (-B \phi / 2)$, with $B=B_{-}+B_{+}$where $B_{-}$and $B_{+}$are commuting normalized 2-blades. We reiterate that this is not equivalent to rewriting the rotor as the exponential of a single 2-blade independent of its starting point; that cannot be done.

We will need a result from the Taylor expansion of a rotor (not necessarily simple) acting on a vector, in terms of its bivector $A$ (from [4], Chapter 7):

$$
e^{-A / 2} x e^{A / 2}=x+(x \cdot A)+\frac{1}{2 !}((x \cdot A) \cdot A)+\frac{1}{3 !}(((x \cdot A) \cdot A) \cdot A)+\cdots
$$

For simple rotors based on a 2-blade $A$, this series can be expressed in terms of trigonometric functions (when $A^{2}<0$ ), hyperbolic functions (when $A^{2}>0$ ) or linear functions (when $A^{2}=0$ ), as we showed in the previous section. The expansion then simplifies to the closed expression Eqs. (3), (4) or (5). However, in our case the rotor is not simple (since our bivector $B$ is not a 2-blade), so we need to proceed more carefully.

We can use Eq. (6) for a small bivector $A=\epsilon B$ to understand the local orbit. The tangent vector to the orbit at $x$ is:

$$
T(x)=\lim _{\epsilon \rightarrow 0} x \wedge(x+(x \cdot \epsilon B)) / \epsilon=x \wedge(x \cdot B),
$$



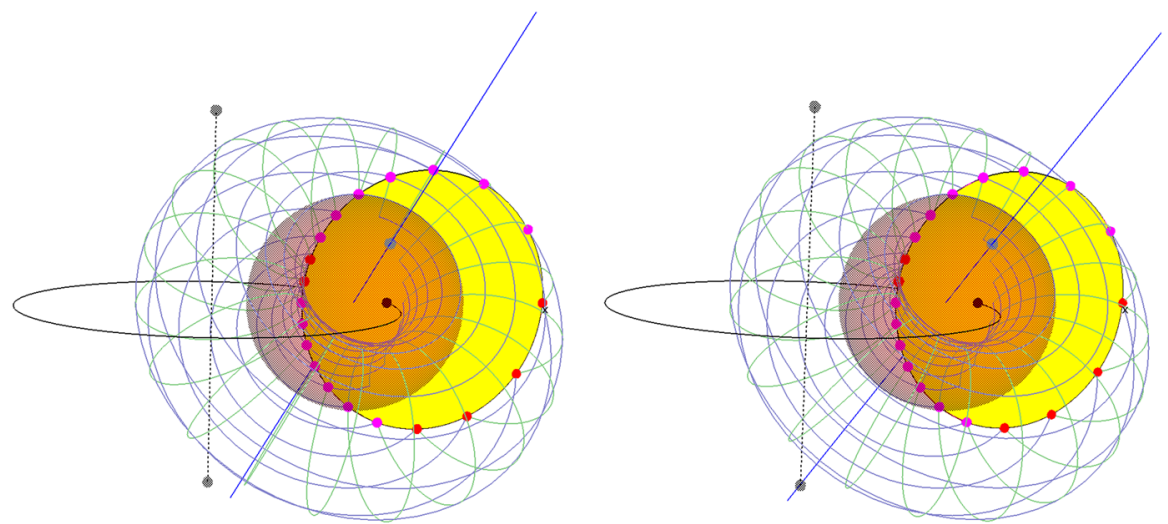

Figure 7. A Villarceau circle generated from a point $x$ in the motion field of the rotor $R_{-} R_{+}$. The 2-blades of the rotor are indicated as a dual line (solid blue), and an imaginary point pair on it (in dashed blue). Along its stepped orbit, $x$ hits parallel and meridian circles $x \wedge B_{+}$(blue) and $x \wedge B_{-}$ (green) on a torus. The Villarceau circle is the border of the yellow disk. The local 2-blade $B_{V}(x)$ of this motion is indicated as the two endpoints of the dotted black line segment; its circle $B_{V}(x)^{*}$ (black) cuts the yellow disk orthogonally in its 'conformal center' (black). The dotted imaginary sphere is $e_{-}$, which is cut by the Villarceau circle in two antipodal points. Two views generated by GAViewer [7], in a cross-eyed stereo pair (color figure online)

while the local osculating circle is:

$$
\begin{aligned}
C(x) & =\lim _{\epsilon \rightarrow 0} x \wedge(x+x \cdot \epsilon B) \wedge\left(x+x \cdot \epsilon B+\frac{1}{2 !}(x \cdot \epsilon B) \cdot \epsilon B\right) / \epsilon^{3} \\
& =x \wedge(x \cdot B) \wedge((x \cdot B) \cdot B) .
\end{aligned}
$$

In our case, the bivector $B$ equals $B=\mathbf{e}_{1} \mathbf{e}_{2}+\mathbf{e}_{3} e_{+}$. For that particular rotor, the tangent circle is the actual full orbit. To show this, we compute the next term in the Taylor series of Eq. (6):

$$
\begin{aligned}
B & =\mathbf{e}_{1} \mathbf{e}_{2}+\mathbf{e}_{3} e_{+} \\
x \cdot B & =\left(x \cdot \mathbf{e}_{1}\right) \mathbf{e}_{2}-\left(x \cdot \mathbf{e}_{2}\right) \mathbf{e}_{1}+\left(x \cdot e_{+}\right) \mathbf{e}_{3}-\left(x \cdot \mathbf{e}_{3}\right) e_{+} \\
(x \cdot B) \cdot B & =-\left(x \cdot \mathbf{e}_{1}\right) \mathbf{e}_{1}-\left(x \cdot \mathbf{e}_{2}\right) \mathbf{e}_{2}-\left(x \cdot \mathbf{e}_{3}\right) \mathbf{e}_{3}-\left(x \cdot e_{+}\right) e_{+} \\
& =-x-\left(x \cdot e_{-}\right) e_{-} \\
((x \cdot B) \cdot B) \cdot B & =-x \cdot B+0 .
\end{aligned}
$$

After three terms, we are back at the first (with a minus sign); therefore the rest of the terms follows the same pattern. When substituting these results in the Taylor series Eq. (6), we can collate terms to express the series exactly on a basis of three elements, as:

$$
e^{-\epsilon B / 2} x e^{\epsilon B / 2}=-e_{-}\left(x \cdot e_{-}\right)+\left(x+\left(x \cdot e_{-}\right) e_{-}\right) \cos \epsilon+(x \cdot B) \sin \epsilon .
$$


This is very similar to the general form of the orbit of an imaginary unit 2-blade as given in Eq. (3):

$$
x(\phi)=(x \wedge P) / P+(x \cdot P) / P \cos \phi+(x \cdot P) \sin \phi .
$$

We observe that in the general form we can establish the original 2-blade of the rotor from the ratio of the factors of sine and cosine. Therefore in our case, the unit 2-blade generating the motion along the Villarceau circle is:

$$
\begin{aligned}
B_{V}(x) & =\left(x+\left(x \cdot e_{-}\right) e_{-}\right)^{-1}(x \cdot B) \\
& =\left(x+\left(x \cdot e_{-}\right) e_{-}\right) \wedge(x \cdot B) /\left(x \cdot e_{-}\right)^{2},
\end{aligned}
$$

since due to $e_{-} \cdot(x \cdot B)=0$ and $x \cdot(x \cdot B)=0$, the geometric product can be replaced by an outer product. The factor $x+\left(x \cdot e_{-}\right) e_{-}$is a real sphere, with center at $2 \mathbf{x} /\left(1-\mathbf{x}^{2}\right)$ and with radius $\left(1+\mathbf{x}^{2}\right) /\left(1-\mathbf{x}^{2}\right)$ (where $\mathbf{x}$ is the Euclidean location of the conformal point $x)$.

With $B_{V}(x)$ as its local 2-blade, the orbit at $C(x)$ passing through $x$ is therefore the circle.

$$
C(x)=x \wedge B_{V}(x) \propto x \wedge(x \cdot B) \wedge e_{-} .
$$

Because of the final rewriting, it would seem that it should be simpler to use instead of $B_{V}(x)$ the 2-blade proportional to $B_{V}^{\prime}(x)=e_{-} \wedge(x \cdot B)$, which has the same orbit $C(x) \propto x \wedge B_{V}^{\prime}(x)$. However, $B_{V}^{\prime}(x)$ is a real point pair, with a source and a sink, and the parametric (time) dependency it generates along its orbit is therefore completely different (as we derived in Sect. 3). So if the parametrization is relevant, we should use $B_{V}$ rather than $B_{V}^{\prime}$; but for properties of the orbit as a whole (such as in the EM field lines of [2]), the simpler $B_{V}^{\prime}$ would be sufficient.

We conclude that the motion performed by $x$ under the rotor with bivector $B$ is equivalent to a pure conformal rotation induced by the imaginary point pair $B_{V}(x)$, and that it generates the Villarceau circle. This point pair $B_{V}(x)$ depends on $x$ itself, so the motion by the rotor $\exp (B / 2)$ is not globally equivalent to a single conformal rotation.

\section{Geometric Properties}

Since the Villarceau circles are orbits of a conformal rotor, they cut the parallels $\left(x \wedge B_{-}\right)$and meridians $\left(x \wedge B_{+}\right)$of the torus at a constant angle. This property is illustrated for the $(1,1)$ knot in Fig. 8. As a consequence of being conformal, all constructions and statements transform covariantly under conformal transformations. Not only can we translate, rotate and scale them from the standard situation, but using spherical inversion we can produce fully analogous properties of Villarceau circles on Dupin cyclides, as we will see in Sect. 7. For now we prefer to continue to use the torus vocabulary, as our token example of these conformally equivalent extensions. For the same reason, we use the unit radius sphere $e_{+}$in $B_{+}=\mathbf{e}_{3} e_{+}$; other scales can be produced by the usual conformal scaling rotors. The covariance of the conformal rotor formalism makes those extensions unnecessary to prove - they are automatic. 


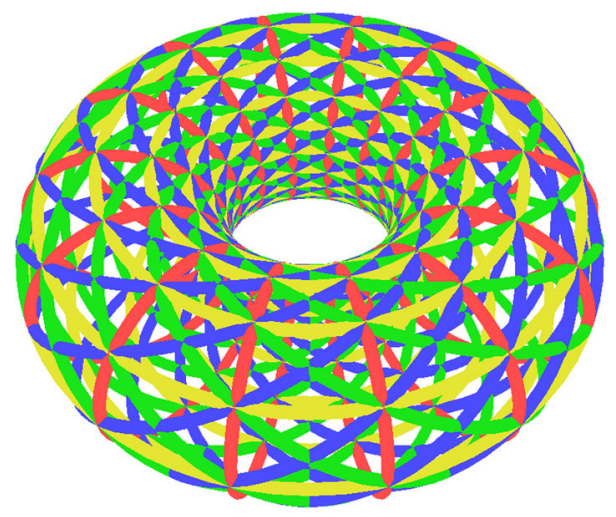

Figure 8. Meridians (in red), parallels (in yellow) and the two types of Villarceau circles (in blue and green) on a torus. (color figure online)

The other Villarceau circle through a point $x$ is generated by reversing one of the rotors, say using $R_{+} \widetilde{R_{-}}$, the exponential of the bivector $B_{+}-$ $B_{-}$rather than $B_{+}+B_{-}$. The situation in Fig. 1 is rather special: there apparently exist points in which the two Villarceau circles passing through them are coplanar. We can easily establish where those points are, as follows. We demand that the two Villarceau circles $x \wedge e_{-} \wedge\left(x \cdot\left(B_{+}+B_{-}\right)\right)$and $x \wedge e_{-} \wedge\left(x \cdot\left(B_{+}-B_{-}\right)\right)$are coplanar (we can now use their simplified form with $e_{-}$only, since their parametrization is immaterial to this property). This implies:

$$
\begin{aligned}
0 & =x \wedge\left(x \cdot\left(B_{+}+B_{-}\right)\right) \wedge\left(x \cdot\left(B_{+}-B_{-}\right)\right) \wedge e_{-} \wedge \infty \\
& =-x \wedge\left(x \cdot B_{+}\right) \wedge\left(x \cdot B_{-}\right) \wedge e_{-} \wedge e_{+} \\
& =\mathbf{x} \wedge\left(\mathbf{x} \cdot\left(\mathbf{e}_{1} \mathbf{e}_{2}\right)\right) \wedge\left(0+\left(x \cdot e_{+}\right) \mathbf{e}_{3}\right) \wedge e_{-} \wedge e_{+} \\
& =\left(x \cdot e_{+}\right)\left(\left(\mathbf{x} \cdot \mathbf{e}_{1}\right)^{2}+\left(\mathbf{x} \cdot \mathbf{e}_{2}\right)^{2}\right) \mathbf{e}_{1} \wedge \mathbf{e}_{2} \wedge \mathbf{e}_{3} \wedge e_{-} \wedge e_{+}
\end{aligned}
$$

(remember that $\mathbf{x}$ is the Euclidean part of the conformal point $x$ ). A trivial non-zero solution is $x=\operatorname{CGA}\left(\alpha \mathbf{e}_{3}\right)$ for any $\alpha$, when the circles both degenerate to a line. But more interesting is the set of points such that $x \cdot e_{+}=0$. Thus in our standard setup, any point $x$ on the real unit sphere $e_{+}$produces a planar pair of Villarceau circles.

We then have to show that all Villarceau circles can be generated from a point on this unit sphere. And indeed they can: the meet of a Villarceau circle with the unit sphere at the origin is the point pair $e_{+} \cdot\left(x \wedge(x \cdot B) \wedge e_{-}\right)$, of which the square is $\left(\frac{\mathbf{x}^{2}+1}{2}\right)^{2}\left(\left(\frac{\mathbf{x}^{2}-1}{2}\right)^{2}+\left(\mathbf{x} \cdot \mathbf{e}_{3}\right)^{2}\right)$. This is non-negative, so the point pair is real (with the only null degeneracy on the core circle of the set of Villarceau circles, where $\mathbf{x}^{2}=1$ and $\mathbf{x} \cdot \mathbf{e}_{3}=0$ ). Thus each Villarceau circle intersects the unit sphere, and each can therefore be generated from such an intersection point. In view of the pairing of Villarceau circles, starting them from a such point on the unit sphere $e_{+}$is perhaps the most natural way to generate the circles in our standardized setup. Of course every circle 
intersects the unit sphere in two points, so in fact only half of the unit sphere is required; though we might use the other half to index Villarceau circles of opposite orientation.

We can obtain some more insight in the geometry of a Villarceau circle by substituting the series expansion terms of Eq. (9). Thus when used in the outer product, the second order term simplifies to

$$
C(x) \propto x \wedge(x \cdot B) \wedge e_{-}=T(x) \wedge e_{-} .
$$

This implies that the Villarceau circle can also be defined as 'tangent to the local tangent vector $T(x)$ and intersecting the unit sphere at the origin in two antipodal points' (since it contains the imaginary unit sphere $e_{-}$as a factor, see [4], Chapter 15 on the 'plunge' operation). This fact is visible in Fig. 7.

Actually, the circle $C(x)$ is invariant under reflection in the imaginary sphere $e_{-}$. To show this, first note that $B$ changes sign under reflection in $e_{-}$since $-e_{-} B / e_{-}=-B$, and that $-e_{-} x / e_{-}=e_{-} x e_{-}=x+2\left(x \cdot e_{-}\right) e_{-}$. Then, rather surprisingly, we find that the sphere $x \cdot B$ (a sphere passing through $x$ and orthogonal to the local tangent vector $T(x))$ is invariant under the reflection:

$$
-e_{-}(x \cdot B) e_{-}^{-1}=e_{-}(x \cdot B) e_{-}=(x \cdot B)+2\left((x \cdot B) \cdot e_{-}\right) e_{-}=x \cdot B,
$$

since $(x \cdot B) \cdot e_{-}=0$. Then we can establish that $C(x)$ is invariant modulo a $\operatorname{sign}$

$$
\begin{aligned}
e_{-} C(x) e_{-} & =-\left(e_{-}(x \wedge(x \cdot B)) e_{-}\right) \wedge e_{-} \\
& =-\left(x+2\left(x \cdot e_{-}\right) e_{-}\right) \wedge(x \cdot B) \wedge e_{-} \\
& =-x \wedge(x \cdot B) \wedge e_{-} \\
& =-C(x)
\end{aligned}
$$

In more detail, the inversion in $e_{-}$exchanges the point $x$ with a point $x^{\prime}$ on the other side of the conformal center of the Villarceau circle (i.e. one of the locations where the real circle $\left(B_{V}\right)^{*}$ intersects the plane $\left.C(x) \wedge \infty\right)$, along an orthogonal circle passing through that conformal center and $x$. The resulting points $x$ and $x^{\prime}$ are symmetric relative to the origin: $x \wedge e_{+} \wedge x^{\prime}$ is a straight line.

Inversion in an imaginary sphere like $e_{-}$is easy to visualize: it is equivalent to an inversion in a real sphere of the same radius and center, followed by a point reflection in that center. For the origin-centered unit spheres of our standard situation, this follows from $e_{-}=\left(e_{-} \wedge e_{+}\right) e_{+}$. When read as a compound operator for sandwiching, the factor $e_{-} \wedge e_{+}=o \wedge \infty$ is the flat point at the origin producing the point reflection. It is not a rotor, yet it can be used in the sandwiching product, see [4] Chapter 16. 


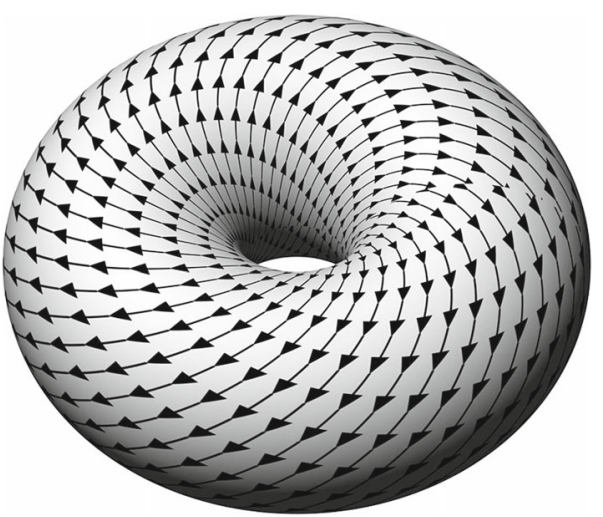

Figure 9. (Graphics and text from wikipedia page: Rotations in 4-dimensional space.) A 4D Clifford torus stereographically projected into 3D looks like a torus, and a double rotation can be seen as a helical path on that torus. For a rotation whose two rotation angles form a rational number, the paths will eventually reconnect, while for an irrational ratio they will not. An isoclinic rotation will form a Villarceau circle on the torus, while a simple rotation will form a circle parallel or perpendicular to the central axis

\section{Hopf Fibration and Stereographic Projection from 4D}

\subsection{Space Filling Villarceau Circles}

In the field generated by the rotor $B$, every point $x$ picks out a Villarceau circle on a torus as its orbit. The torus is determined by the circles $x \wedge B_{-}$ and $x \wedge B_{+}$, and the Villarceau circle is $x \wedge B_{V}(x)$, with $B_{V}(x)$ defined as in Eq. (11). Clearly the points of 3D space can be grouped in equivalence classes of the circles of points that are on the same Villarceau circle generated by $B_{V}(x)$; this class may be indexed by a point on the unit sphere $e_{+}$(in a double cover, or in two opposite orientations). All Villarceau circles on the same torus are a larger equivalence class, rotationally invariant under the rotor $R_{-}$, see Fig. 9, and each indexed by a point on a shared parallel of the unit sphere. Moving the index point $x$ to other classes generates Villarceau circles on a different torus, involving the conformal rotation $R_{+}$.

All in all, a space-filling set of circles appears, together forming a Hopf fibration of 3-dimensional space, see Fig. 10. There are two singular tori; one consists of the single circle $\left(B_{+}\right)^{*}$ (the conformal center circle of any of the tori in the fibration), the other consists of the single line $\left(B_{-}\right)^{*}$ (the axis of any torus in the fibration).

\subsection{Isoclinic Rotations in $4 \mathrm{D}$}

As we have seen, there are two rotors involved in composing the rotations, in our specific example produced by the commuting 2 -blades $B_{-}=\mathbf{e}_{1} \mathbf{e}_{2}$ and $B_{+}=\mathbf{e}_{3} e_{+}$. Since $e_{+}^{2}=1$, we could consider the four vectors involved as just 

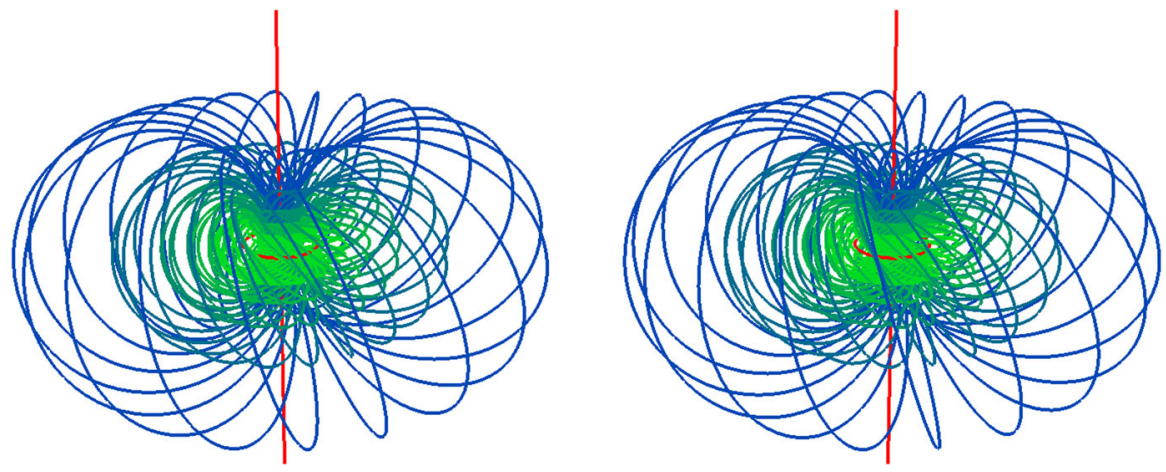

Figure 10. Some of the linked and nested Villarceau circles on nested tori in the Hopf fibration, in a cross-eyed stereo pair. The two exceptional Hopf limit circles are indicated in red (color figure online)

an orthonormal basis of a 4-dimensional space. Let us make this explicit by replacing $e_{+}$by $\mathbf{e}_{4}$. Then the 4 -dimensional space we have constructed for those two rotations is a Clifford torus (the product of two circular motions).

In that 4-dimensional space, a general rotation has two planes in which it needs to be specified, and we have chosen them orthogonally. But for the special rotor with 2 -blade $B=B_{+}+B_{-}$, those rotations are coupled and performed at the same rate. This composite motion is called an isoclinic rotation, and normally one would consider it primarily in the 4D Euclidean space spanned by four orthogonal unit basis vectors $\left\{\mathbf{e}_{1}, \mathbf{e}_{2}, \mathbf{e}_{3}, \mathbf{e}_{4}\right\}$.

Our algebraically equivalent representation on the basis $\left\{\mathbf{e}_{1}, \mathbf{e}_{2}, \mathbf{e}_{3}, e_{+}\right\}$ also consists of four orthogonal unit vectors (though the full basis contains an additional negatively squared vector $e_{-}$), but now all can be drawn in 3D (as the three coordinate planes plus the unit sphere, all at the origin). Doing so apparently shows a toroidal image of the unit 3-sphere in $4 \mathrm{D}$, converting the orbits of 3D points under an isoclinic rotation (which reside on that sphere) into the set of circles of the Hopf fibration.

In fact, our replacing $e_{+}$by $\mathbf{e}_{4}$, which algebraically looks like a simple relabeling, is geometrically equivalent to performing an inversion in a sphere, of the $4 \mathrm{D}$ space. And the selection of a 3D subspace of the result makes this into a stereographic projection, from the unit sphere in $4 \mathrm{D}$ to a flat $3 \mathrm{D}$ space. These known facts take on a simple computational form in CGA, as follows.

To perform the desired stereographic projection from $4 \mathrm{D}$ to $3 \mathrm{D}$, consider the dual sphere $e_{+}$; it resides in either space. Use $\sigma=\mathbf{e}_{4}+e_{+}$(the real dual sphere in $4 \mathrm{D}$ with center at $\mathbf{e}_{4}$ and radius $\sqrt{2}$ ) in the sandwich product $X \mapsto-\frac{1}{2} \sigma X \sigma$. Then the non-involved basis vectors are invariant:

$$
\mathbf{e}_{1} \mapsto \mathbf{e}_{1}, \quad \mathbf{e}_{2} \mapsto \mathbf{e}_{2}, \quad \mathbf{e}_{3} \mapsto \mathbf{e}_{3} \quad \text { (and } \quad e_{-} \mapsto e_{-} \text {) }
$$

but $\mathbf{e}_{4}$ and $e_{+}$are swapped:

$$
\mathbf{e}_{4} \mapsto e_{+}, \quad e_{+} \mapsto \mathbf{e}_{4} .
$$




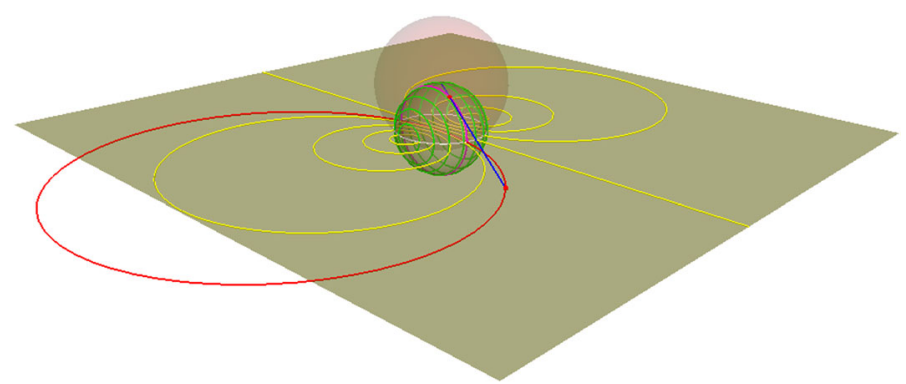

Figure 11. Stereographic projection from 3D to 2D, by inversion in the sphere $\mathbf{e}_{3}+e_{+}$(in light red) converts the green unit sphere $e_{+}$into the yellow plane $\mathbf{e}_{3}$. The green circles (orbits of $\exp \left(-\phi \mathbf{e}_{1} \mathbf{e}_{3} / 2\right)$ for a point on the unit sphere) become yellow circles in the plane, orbits of $\exp \left(-\phi \mathbf{e}_{1} e_{+} / 2\right)$ for the stereographic projection of that point (color figure online)

As a consequence, orbits on the unit sphere $e_{+}$in 4 -space of, say, the 4D Euclidean rotation with 2 -blade $\mathbf{e}_{3} \mathbf{e}_{4}$ become mapped to circles in $3 \mathrm{D}$, the orbits of a conformal rotation in 3 -space with 2 -blade $\mathbf{e}_{3} e_{+}$. Since this no longer involves $\mathbf{e}_{4}$, we can depict the results in a (conformal) $3 \mathrm{D}$ space. The inversion in $\sigma$ also maps the rest of the $4 \mathrm{D}$ space (i.e. points not on its unit sphere), to points outside the hyperplane $\mathbf{e}_{4}$. We do not depict those when interpreting the mapping as a stereographic projection. Thus only the sphere $S^{3}$ (i.e. $e_{+}$in $4 \mathrm{D}$ ) is depicted in compactified $\mathbb{R}^{3}$ (which is the space that constitutes all points of the $4 \mathrm{D}$ coordinate plane $\mathbf{e}_{4}$, plus the point at infinity).

In an attempt to illustrate this by analogy, in Fig. 11 we show the same principle in one dimension less. We map from $3 \mathrm{D}$ to $2 \mathrm{D}$, employing the versor $e_{+}+\mathbf{e}_{3}$ to swap the 2 -sphere $e_{+}$of $3 \mathrm{D}$ with the plane $\mathbf{e}_{3}$, which is then interpreted as a compactified $2 \mathrm{D}$ space.

To express and derive certain properties of Villarceau circles very compactly, we have seen that the orthonormal basis of our algebra needs to be completed by the imaginary sphere $e_{-}$. The existence of this 5 -sphere basis for 3D space was already explored in a 1934 paper by Raynor [11]. The imaginary sphere $e_{-}$is not affected by the inversion above, and so can be considered to be part of both the $4 \mathrm{D}$ and the $3 \mathrm{D}$ spaces involved in our construction.

\section{Villarceau Circles on Dupin Cyclides}

Within CGA, it is easy to generalize from the specific unit-sphere and unitplane based generators we have employed in this paper. The conformal covariance of all expressions implies that we may apply any conformal versor $V$ to all elements, and preserve their relationships. The corresponding 2-blades 


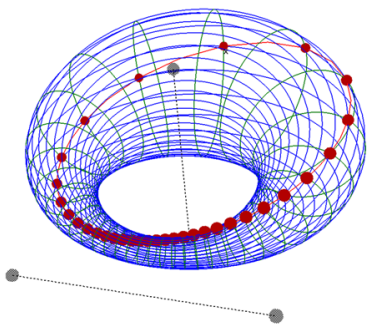

(a)

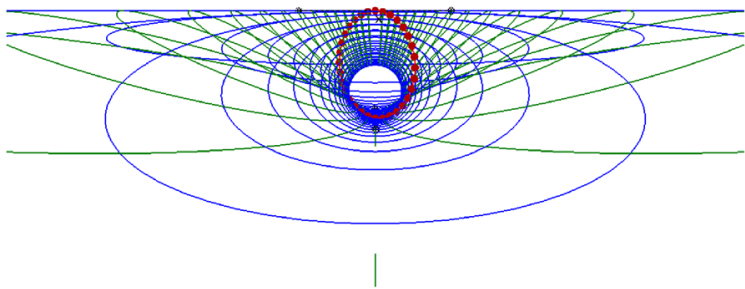

(b)

FiguRE 12. a A Villarceau circle on a cyclide and b a Villarceau circle on a degenerate unbounded cyclide. The two imaginary point pairs generating the cyclides are indicated as the grey halters

$B_{-}=\mathbf{e}_{1} \mathbf{e}_{2}$ and $B_{+}=\mathbf{e}_{3} e_{+}$change into more general expressions $V B_{+} / V$ and $V B_{-} / V$ involving non-unit-radius spheres. The role of $e_{-}$in our analysis is played by the imaginary sphere $V e_{-} / V$.

In fact, there is not much more to say about this, since obviously any property that can be formulated in a covariant manner transforms covariantly. So we merely show some Villarceau circles on cyclides in Fig. 12 that are the equivalent (by conformal transformations) of Fig. 3b for the torus.

We just have to be a bit careful transferring the property of Fig. 1: namely that the two Villarceau circles can be considered as a planar cross section of a torus. The original cut plane of the figure will transform to a sphere under the conformal versor, and it is a trivial statement that two 3D circles intersecting in a point pair lie on a common sphere. So the desired cut plane of the cyclide is not the transform of the cut plane of the torus. The proper procedure is to transfer not the plane itself, but the condition of Eq. (12): that for each Villarceau circle generated by $B_{+}+B_{-}$there exists a point $x$ on it so that the circle generated by $B_{+}-B_{-}$from that point shares a plane with the first circle. The non-trivial solution for our standard situation was $x \cdot e_{+}=0$ : we can start any Villarceau circle and its coplanar counterpart from the same point on the unit sphere. After the conformal transformation, that condition becomes $x^{\prime} \cdot\left(V e_{+} / V\right)=0$ for transformed points $x^{\prime}$. Points on this transformed sphere still produce two Villarceau circles in a coplanar pair. Therefore the cyclides, too, can be cut by a well-chosen plane to produce two intersecting Villarceau circles. Figure 13 illustrates this fact, for an interesting special case in which one of the Villarceau circles has become a line.

\section{Maxwell-Dirac Toroids}

As stated in the introduction, Villarceau circles occur in physics. When they are specific field lines related to the electromagnetic field in vacuum, as in [2], they are geometric objects without a need for a parametrization. Such field lines themselves move with the speed of light, and are space filling in 


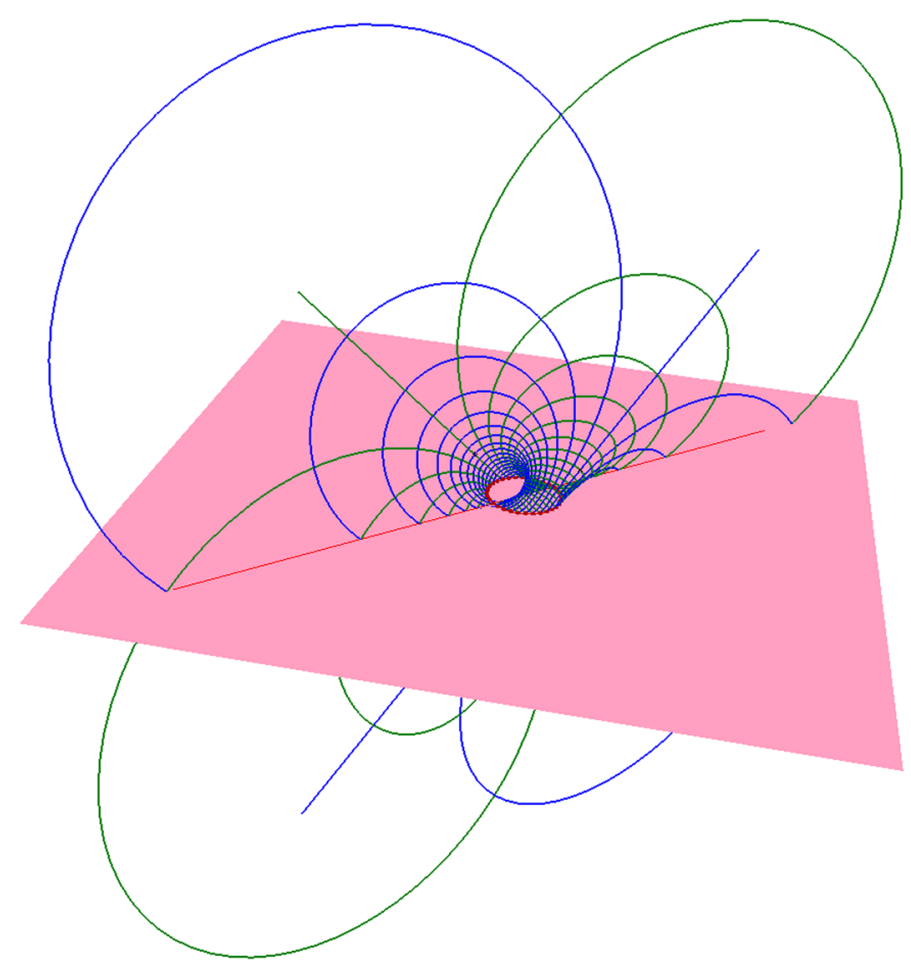

FiguRE 13. An unbounded cyclide (generated from the same imaginary point pair generator as in Fig. 12a by a well-chosen spatial point). Two Villarceau circles are indicated in red - one has degenerated to a line - as well as the cutting plane that can be thought to produce them (color figure online)

the manner of a Hopf fibration. The electric field $\mathbf{E}$ and the magnetic field $\mathbf{B}$ deform under this motion (or similarly, if they are considered in a different inertial frame of reference), though their Poynting field line $\mathbf{E} \times \mathbf{B}$ retains its canonical shape of a Villarceau circle in a Hopf fibration. Our way of describing this geometry compactly by a location-dependent circle may be of some interest in that field. It is well known that Maxwell's equation exhibits conformal symmetries (see e.g. [12]), so one would expect conformal rotor descriptions of the geometry it generates to be efficacious. The conformal Villarceau orbits from this paper are a special case of the more general conformal $(m, n)$ knots treated in [5], which may then apply to the more general fields, also exposed in [2].

As for the toroidal motion of the electron (and the hypothesized internal structure of the photon), [9] derives the Consa parametrization of the torus directly from the Dirac equation. To do so, Hestenes employs a factorization of the spinor wave function that splits off a Lorentz boost, leaving a purely 
Euclidean rotor of two factors that easily give rise to the Consa toroidal solution, in the Euclidean two-angle parametrization by what we would call simple rotors with 2-blades $\mathbf{e}_{1} \mathbf{e}_{2}$ and $\mathbf{e}_{2} \mathbf{e}_{3}$. That sequence of Euclidean rotations makes the Consa parametrization of the torus seem natural, though the parameter is not physical travel time - the travel speed is actually hypothesized to be uniform (equal to the speed of light). We suggest that factorizing the wave function into conformal components (in our parlance, the rotors of $\mathbf{e}_{1} \mathbf{e}_{2}$ and $\left.\mathbf{e}_{3} e_{+}\right)$may also be feasible, and in that context the parametrization from this paper may be useful. This is not just a change of representation: should the electron move in a closed $(1,1)$ knot motion along the torus, then the geometry of the spatial orbit differs between the uniform and the conformal parametrizations. The conformal parametrization would have it moving in a plane (as we showed in this paper), but the uniform angle parametrization of the doubly-periodic motion of the Consa model is non-planar. The different accelerations those two motions impose may lead to physically observable consequences, which would force the natural choice between them. Motion along the general $(m, n)$ knots would similarly be geometrically, and hence physically, different.

The conformal description of the Robinson congruence (the guise of Villarceau circles in twistor theory) has been given in terms of conformal rotors in [1], for massless particles. Viewing the Villarceau circles of the congruence as a special case of $(m, n)$ conformal torus knots may perhaps allow extension of that geometrical twistor theory to treat other particles as well.

Open Access. This article is distributed under the terms of the Creative Commons Attribution 4.0 International License (http://creativecommons.org/licenses/by/4. $0 /$ ), which permits unrestricted use, distribution, and reproduction in any medium, provided you give appropriate credit to the original author(s) and the source, provide a link to the Creative Commons license, and indicate if changes were made.

Publisher's Note Springer Nature remains neutral with regard to jurisdictional claims in published maps and institutional affiliations.

\section{References}

[1] Arcaute, E., Lasenby, A., Doran, C.: A representation of twistors within geometric (Clifford) algebra. Adv. Appl. Clifford Algebra 18, 373-394 (2008)

[2] Arrayás, M., Bouwmeester, D., Trueba, J.L.: Knots in electromagnetism. Phys. Rep. 667, 1-61 (2017)

[3] Consa, O.: Helical solenoid model of the electron. Progress Phys. 14(2), 80-89 (2018)

[4] Dorst, L., Fontijne, D., Mann, S.: Geometric Algebra for Computer Science: An Object-oriented Approach to Geometry. Morgan Kaufman, Massachusetts (2009)

[5] Dorst, L.: The construction of 3D conformal motions. Math. Comput. Sci. 10(1), 97-113 (2016) 
[6] Fontijne, D., Dorst, L.: Reconstructing rotations and rigid body motions from exact point correspondences through reflections. In: Dorst, L., Lasenby, J. (eds.) Guide to Geometric in Practice, pp. 63-78. Springer, New York (2011)

[7] Fontijne, D., Dorst, L.: GAViewer, free download for various platforms. http://www.geometricalgebra.net/gaviewer_download.html (2007). Accessed Jan 2019

[8] Hestenes, D.: Deconstructing the electron clock. Published on the AGACSE 2018 website https://www.ime.unicamp.br/ agacse2018/abstracts/ InvitedSpeakers/Hestenes-Maxwell-Dirac.pdf (2018). Accessed Jan 2019

[9] Hestenes, D.: Maxwell-Dirac electron theory (in preparation) (2019)

[10] Needham, T.: Visual Complex Analysis. Clarendon Press, Oxford (1997)

[11] Raynor, G.E.: On $n+2$ mutually orthogonal hyperspheres in Euclidean $n$-space. Am. Math. Monthly 41(7), 424-438 (1934)

[12] Rosen, G.: Conformal invariance of Maxwell's equations. Am. J. Phys. 40, 1023 (1972)

[13] Wikipedia. Villarceau circles. https://en.wikipedia.org/wiki/Villarceau_circles (2018). Accessed Jan 2019

Leo Dorst

Computer Vision Group

Informatics Institute University of Amsterdam

Amsterdam

The Netherlands

e-mail: 1.dorst@uva.nl

Received: January 28, 2019.

Accepted: April 9, 2019. 